\title{
Design validation of the gyrotron diamond output window for the upgrade of the ECRH system at W7-X
}

G. Aiello1, K.A. Avramidis², G. Gantenbein², J. Jelonnek², J. Jin², H.P. Laqua ${ }^{3}$, A. Meier ${ }^{1}$, T. Scherer ${ }^{1}$, D. Strauss ${ }^{1}$, M. Thumm² $^{2}$ IIAM, ${ }^{2}$ IHM, Karlsruhe Institute of Technology (KIT), 76344 Eggenstein-Leopoldshafen, Germany / ${ }^{3}$ Max Planck Institute for Plasma Physics (IPP), 17491 Greifswald, Germany

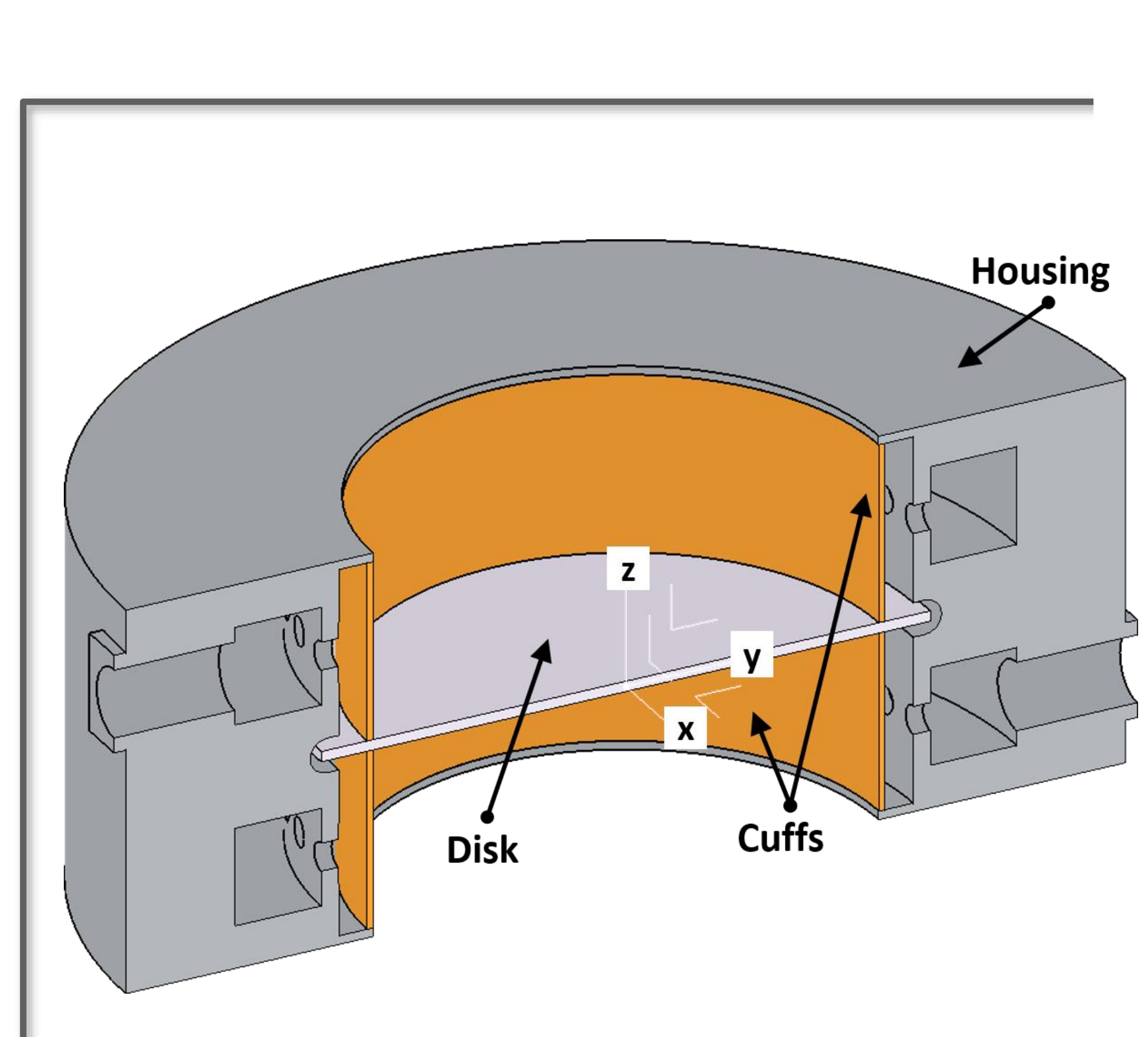

Motivation

The W7-X gyrotron design, operating successfully at $1 \mathrm{MW}$ and $140 \mathrm{GHz} \mathrm{CW}$, is being upgraded for operating at $1.5 \mathrm{MW}$ to achieve regimes with high plasma beta and low collisionality

The gyrotron features a CVD diamond output window with a $1.8 \mathrm{~mm}$ thick and $106 \mathrm{~mm}$ diameter diamond disk brazed to two copper cuffs, which are enclosed in a stainless steel housing

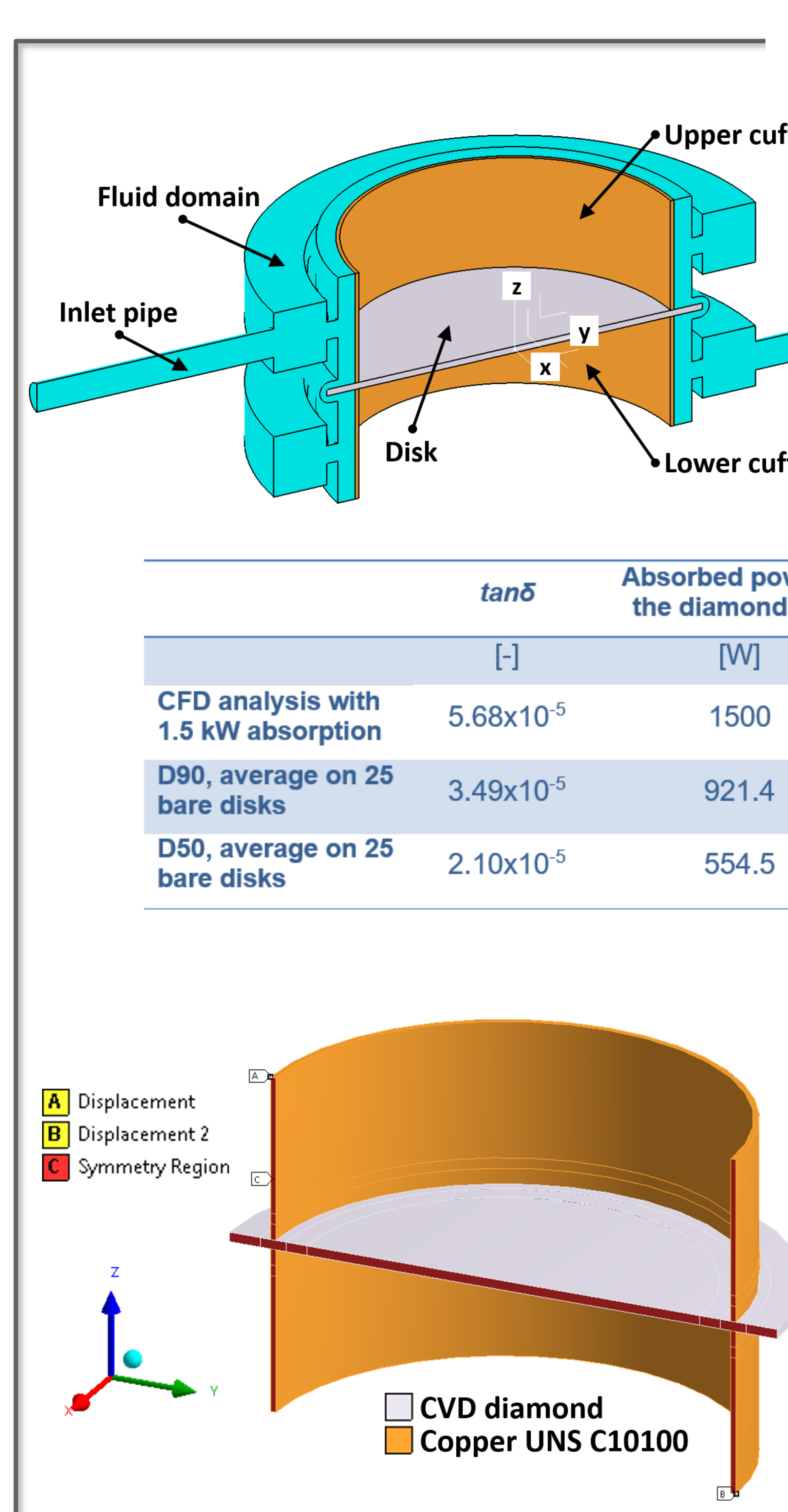

\section{Approach}

CFD conjugated heat transfer analysis Symmetry: half of the window modelled

- Coolant: silicon oil Dow Corning 200(R) with the kinematic viscosity $5 \mathrm{cSt}$

Mass flow rate of $0.2 \mathrm{~kg} \mathrm{~s}^{-1}$ at inlet $\left(25^{\circ} \mathrm{C}\right)$ and $0 \mathrm{~Pa}$ reference pressure at outlet

$1.5 \mathrm{MW}$ Gaussian mm-wave beam with $20 \mathrm{~mm}$ radius

- Heat load applied to disk with Gaussian distribution normalized to obtain in the disk the total absorbed power of $1.5 \mathrm{~kW}$ (comparison reasons to $1 \mathrm{MW}$ case analyzed in 2010 with $1 \mathrm{~kW}$ power absorption)

- Runs with different tano values and $\mathrm{mm}$ wave beam radius (15 $\mathrm{mm}$ and $24 \mathrm{~mm}$ )

\section{Structural analysis}

Plastic steady-state structural analysis: multilinear isotropic hardening

Load in terms of temperature distribution

Stress assessment for the two extreme cases: cuffs free to expand (isostatic boundary condition) and expansion blocked by housing (fixed support condition)

This work has been carried out within the framework of the EURofusion Consortium and has received funding from the Euratom research and training
programme 2014-2018 and 2019-2020 under grant agreement No 633053. The views and opinions expressed herein do not necessarily reflect those of the KIT - The Research University in the Helmholtz Association

\section{Objectives}

- Investigate the window performance at $1.5 \mathrm{MW}$ operation from the thermal perspective, in comparison to the $1 \mathrm{MW}$ case, by CFD conjugated heat transfer analyses (ANSYS CFX V19.2)

- Perform sensitivity studies with respect to the mm-wave beam radius at the window location and to the absorbed power in the disk by using loss tangent (tanঠ) values obtained as average over measurements done in the past for 25 bare disks for W7-X at the KIT laboratories

- Validate the window design against the applicable stress limits by structural analyses (ANSYS V19.2)

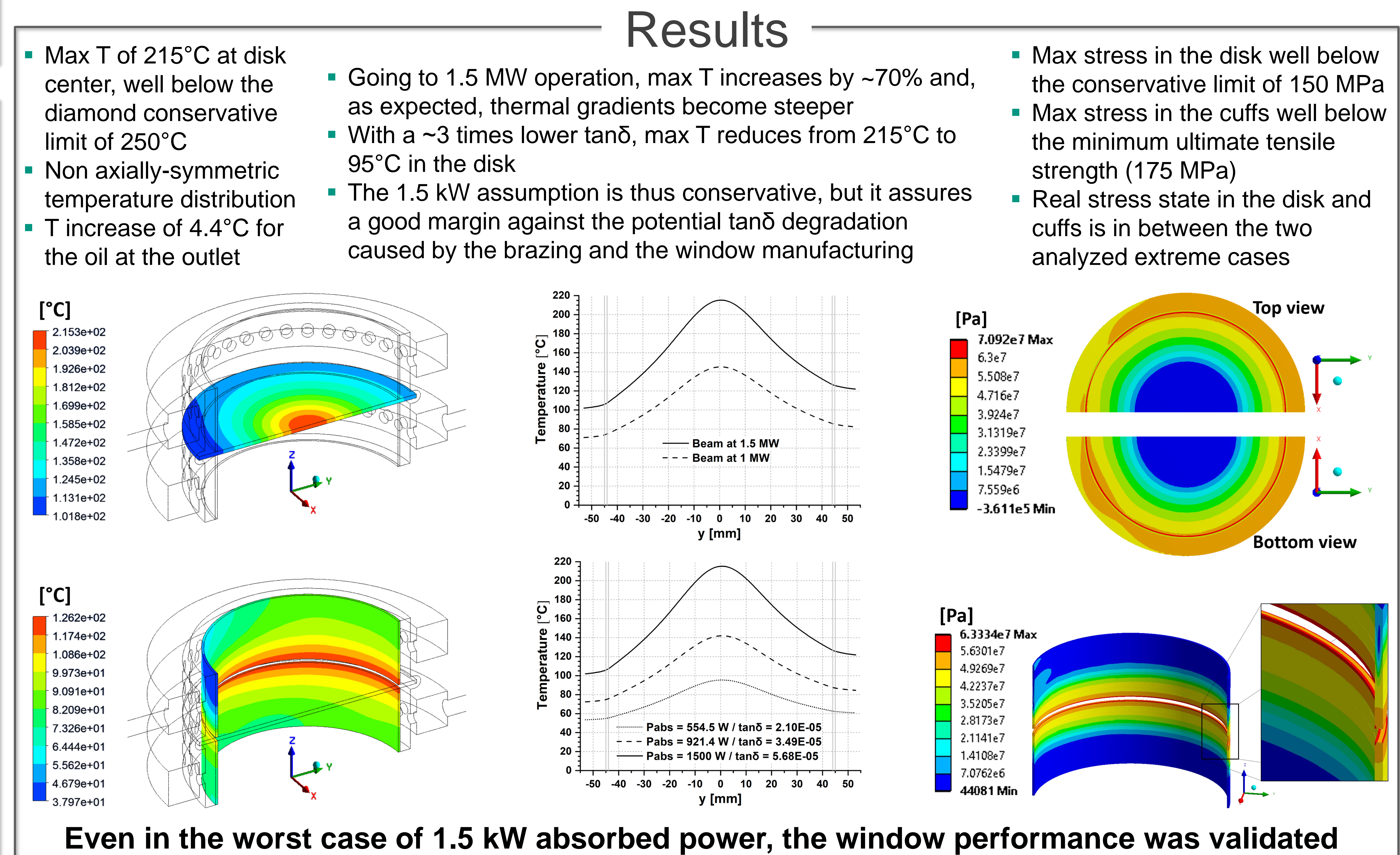

Even in the worst case of $1.5 \mathrm{~kW}$ absorbed power, the window performance was validated

Coordinates for online discussion: $2^{\text {st }}$ of September 2020, 17:00 - 18:30 by Skype (contact: gaetanoaiello1984) Email: gaetano.aiello@kit.edu 